\title{
Unbounded Derivations and Invariant Trace States
}

\author{
Ola Bratteli ${ }^{\star \star \star}$ \\ Centre de Physique Théorique, CNRS Marseille, F-13274 Marseille Cedex 2, France
}

\section{Derek W. Robinson}

Université d'Aix-Marseille II, U.E.R. de Luminy and Centre de Physique Théorique, CNRS Marseille, F-13274 Marseille Cedex 2, France

\begin{abstract}
Let $\mathfrak{M}$ be a von Neumann algebra with cyclic trace vector $\Omega$. Let $\delta(A)=i[H, A]$ be a spatial derivation of $\mathfrak{M}$ implemented by an operator $H$ such that $H \Omega=0$ and $H$ is essentially self-adjoint on $D(\delta) \Omega$.

It follows that:

$e^{i t H} \mathfrak{M} e^{-i t H}=\mathfrak{M}, \quad t \in \mathbb{R}$.
\end{abstract}

\section{Introduction}

In a previous paper [1] we discussed the general theory of unbounded derivations of a von Neumann algebra $\mathfrak{M}$ on a Hilbert space $\mathscr{H}$ and, in particular, introduced the notion of a spatial derivation. This latter form of derivation is defined in terms of a symmetric operator $H$, on $\mathscr{H}$, and a weakly dense *-subalgebra $D(\delta)$ of $\mathfrak{M}$, which leaves the domain $D(H)$ of $H$ invariant. The derivation $\delta$ is defined to be a mapping

$$
A \in D(\delta) \rightarrow \delta(A) \in \mathfrak{M}
$$

with the property that

$$
\delta(A) \psi=i[H, A] \psi, \quad \psi \in D(H) .
$$

It is of particular interest to study the case that $H$ is self-adjoint and has an eigenvector $\Omega$ such that $D(\delta) \Omega$ is a core of $H$. In [1] it was conjectured that if $\Omega$ is also cyclic and separating for $\mathfrak{M}$ then

$$
e^{i t H} \mathfrak{M} e^{-i t H}=\mathfrak{M}, \quad t \in \mathbb{R} .
$$

This conjecture was verified in various special cases. If $\mathfrak{M}$ is abelian then it is essentially a theorem of Gallavotti and Pulvirenti [2]. In this note we extend the abelian result by verifying the conjecture whenever $\Omega$ is a trace vector.

\section{Main Theorem}

Theorem 1. Let $\mathfrak{M}$ be a von Neumann algebra on a Hilbert space $\mathscr{H}$ and let $\Omega$ be a cyclic normalized vector defining a trace on $\mathfrak{M}$, i.e.

$$
(\Omega, A B \Omega)=(\Omega, B A \Omega), \quad A, B \in \mathfrak{M} .
$$

* Supported by the Norwegian Research Council for Science and Humanities.

$\star \star$ Present address: ZIF der Universität Bielefeld, D-4800 Bielefeld, Federal Republic of Germany 
Let $\delta$ be a spatial derivation of $\mathfrak{M}$ implemented by a self-adjoint operator $H$ such that $H \Omega=0$.

If $H$ is essentially self-adjoint on $D(\delta) \Omega$ then

$e^{i t H} \mathfrak{M} e^{-i t H}=\mathfrak{M}, \quad t \in \mathbb{R}$.

The proof of the theorem will be divided into three Lemmas.

Lemma 1. Let $\mathfrak{M}$ be a von Neumann algebra with a normalized cyclic trace vector $\Omega$. Assume that there exists a sequence $B_{n}=B_{n}^{*} \in \mathfrak{M}$ such that $B_{n} \Omega \rightarrow \psi$.

It follows that there exists a self-adjoint operator $B$ affiliated with $\mathfrak{M}$ such that $B_{n} \rightarrow B$ in the strong resolvent sense. In particular if $\chi \in \mathscr{S}(\mathbb{R})$ then $\chi\left(B_{n}\right)$ converges strongly to $\chi(B)$.

Proof. For each $\lambda \in \mathbb{C} \backslash \mathbb{R}$ one has

$$
\begin{aligned}
\|((\lambda & \left.\left.-B_{n}\right)^{-1}-\left(\lambda-B_{m}\right)^{-1}\right) \Omega \| \\
& =\left\|\left(\lambda-B_{n}\right)^{-1}\left(B_{m}-B_{n}\right)\left(\lambda-B_{m}\right)^{-1} \Omega\right\| \\
& \leqq|\operatorname{Im} \lambda|^{-1}\left\|\left(B_{m}-B_{n}\right)\left(\lambda-B_{m}\right)^{-1} \Omega\right\| \\
& =|\operatorname{Im} \lambda|^{-1}\left\|\left(\bar{\lambda}-B_{m}\right)^{-1}\left(B_{m}-B_{n}\right) \Omega\right\| \\
& \leqq|\operatorname{Im} \lambda|^{-2}\left\|\left(B_{m}-B_{n}\right) \Omega\right\|,
\end{aligned}
$$

where we have twice used

$$
\left\|\left(\lambda-B_{n}\right)^{-1}\right\| \leqq|\operatorname{Im} \lambda|^{-1}
$$

and, at the third stage, used the trace property. This demonstrates that the resolvents $\left(\lambda-B_{n}\right)^{-1}$ converge strongly on $\Omega$. But the resolvents are uniformly bounded in $n$ and $\Omega$ is cyclic for the commutant $\mathfrak{M}^{\prime}$ of $\mathfrak{M}$. Hence the resolvents converge strongly to some element $R_{\lambda}$ of $\mathfrak{M}$. We next prove that $R_{\lambda}$ is the resolvent of a self-adjoint operator $B$.

Define $\psi_{n}$ by

$$
\psi_{n}=\left(\lambda-B_{n}\right) \Omega
$$

and hence

$$
\lim _{n \rightarrow \infty} \psi_{n}=\lambda \Omega-\psi .
$$

Now

$$
\begin{aligned}
\| \Omega- & R_{\lambda}(\lambda \Omega-\psi) \| \\
= & \left\|\left(\lambda-B_{n}\right)^{-1} \psi_{n}-R_{\lambda}(\lambda \Omega-\psi)\right\| \\
\leqq & \left\|\left(\lambda-B_{n}\right)^{-1}\left(\psi_{n}-(\lambda \Omega-\psi)\right)\right\| \\
& \quad+\left\|\left(\left(\lambda-B_{n}\right)^{-1}-R_{\lambda}\right)(\lambda \Omega-\psi)\right\| \\
\leqq & |\operatorname{Im} \lambda|^{-1}\left\|\psi_{n}-(\lambda \Omega-\psi)\right\| \\
& \quad+\left\|\left(\left(\lambda-B_{n}\right)^{-1}-R_{\lambda}\right)(\lambda \Omega-\psi)\right\| \underset{n \rightarrow \infty}{\longrightarrow} 0 .
\end{aligned}
$$

Hence one concludes that

$$
R_{\lambda}(\lambda \Omega-\psi)=\Omega .
$$


Thus for $C \in \mathfrak{M}^{\prime}$

$$
\begin{aligned}
R_{\lambda} C(\lambda \Omega-\psi) & =C R_{\lambda}(\lambda \Omega-\psi) \\
& =C \Omega .
\end{aligned}
$$

But as $\Omega$ is cyclic for $\mathfrak{M}^{\prime}$ this demonstrates that the range of $R_{\lambda}$ is dense. By the Kato-Trotter theorem [3] there exists a unique self-adjoint operator $B$ such that

$$
R_{\lambda}=(\lambda-B)^{-1} .
$$

Moreover

$$
e^{i t B_{n}} \psi \rightarrow e^{i t B} \psi
$$

for all $\psi$, uniformly for $t$ in compacts.

Since

$$
(\lambda-B)^{-1}(\lambda \Omega-\psi)=\Omega
$$

one immediately concludes that

$$
B \Omega=\psi \text {. }
$$

Finally for $\chi \in \mathscr{S}(\mathbb{R})$

$$
\chi\left(B_{n}\right) \psi=\int d p e^{i p B_{n}} \psi \hat{\chi}(p)
$$

and

$$
\chi(B) \psi=\int d p e^{i p B} \psi \hat{\chi}(p) .
$$

Hence $\chi\left(B_{n}\right)$ converges strongly to $\chi(B)$.

Lemma 2. Adopt the assumptions of Theorem 1. If $B=B^{*} \in D(\delta)$ and

$$
A=(1+\alpha \delta)(B)
$$

with $\alpha \in \mathbb{R} \backslash\{0\}$ then

$$
(\Omega, \chi(B) B \Omega)=(\Omega, \chi(B) A \Omega)
$$

for all $\chi \in \mathscr{S}(\mathbb{R})$.

Proof. As $A-B=\alpha \delta(B)$ the statement of the Lemma is equivalent to

$(\Omega, \chi(B) \delta(B) \Omega)=0$

for all $\chi \in \mathscr{S}(\mathbb{R})$.

Let $f$ be a function such that $f^{\prime}=\chi$. The Fourier transforms then satisfy

$$
\text { ip } \hat{f}(p)=\hat{f}^{\prime}(p)=\hat{\chi}(p) \text {. }
$$

Thus by Lemma 2 of [4] one has $f(B) \in D(\delta)$ and

$$
\delta(f(B))=i \int_{-\infty}^{\infty} d p p \hat{f}(p) \int_{0}^{1} d r e^{i p r B} \delta(B) e^{i p(1-r) B} .
$$


The trace property of $\Omega$ then yields

$$
\begin{aligned}
(\Omega, \delta(f(B)) \Omega) & =i\left(\Omega, \int_{-\infty}^{\infty} d p p \hat{f}(p) e^{i p B} \delta(B) \Omega\right) \\
& =(\Omega, \chi(B) \delta(B) \Omega) .
\end{aligned}
$$

Hence as $H \Omega=0$ one has

$(\Omega, \chi(B) \delta(B) \Omega)=0$.

Lemma 3. Adopt the assumptions of Theorem 1. If $A=A^{*} \in \mathfrak{M}$ and $\alpha \in \mathbb{R} \backslash\{0\}$ then there exists a self-adjoint $B$ affiliated with $\mathfrak{M}$ such that

$$
B \Omega=(1+i \alpha H)^{-1} A \Omega
$$

and, furthermore,

$$
(\Omega, \chi(B) B \Omega)=(\Omega, \chi(B) A \Omega)
$$

for all $\chi \in \mathscr{S}(\mathbb{R})$.

Proof. As $D(\delta) \Omega$ is a core for $H$ there exists a sequence $A_{n}=(1+\alpha \delta)\left(B_{n}\right)$ such that $A_{n} \Omega$ converges to $A \Omega$. But

$$
\begin{aligned}
A \Omega & =A^{*} \Omega \\
& =\lim _{n \rightarrow \infty} A_{n}^{*} \Omega \\
& =\lim _{n \rightarrow \infty}(1+\alpha \delta)\left(B_{n}^{*}\right) \Omega,
\end{aligned}
$$

where the second step uses the trace property of $\Omega$. Replacing $B_{n}$ by $\left(B_{n}+B_{n}^{*}\right) / 2$ we may assume the $B_{n}$ self-adjoint.

Because

$$
(1+\alpha \delta)\left(B_{n}\right) \Omega=(1+i \alpha H) B_{n} \Omega
$$

and the resolvent of $H$ is bounded we conclude that

$$
B_{n} \Omega=(1+i \alpha H)^{-1} A_{n} \Omega
$$

converges to $(1+i \alpha H)^{-1} A \Omega$. The existence of $B$ now follows from Lemma 1 . Further $B_{n}$ converges to $B$ in the strong resolvent sense.

Next from Lemma 2

$$
\left(\Omega, \chi\left(B_{n}\right) B_{n} \Omega\right)=\left(\Omega, \chi\left(B_{n}\right) A_{n} \Omega\right)
$$

and the desired result follows by limiting.

Proof of Theorem 1. From Theorem 6 of [1] it suffices to show that

$$
(1+i \alpha H)^{-1} \mathfrak{M}_{+} \Omega \subseteq \overline{\mathfrak{M}_{+} \Omega}, \quad \alpha \in \mathbb{R} \backslash\{0\} .
$$

In order to show this take $A \geqq 0$ in Lemma 3 and $\chi$ also positive. One then has

$$
(\Omega, \chi(B) B \Omega)=(\Omega, \chi(B) A \Omega) \geqq 0
$$

by the trace property. Since $\Omega$ is separating for $\mathfrak{M}$ it follows that $\chi(B) B$ can never be negative for $\chi$ positive. Hence $B \geqq 0$ and the proof is complete. 
Remark. As $\Omega$ is a trace vector for $\mathfrak{M}$ it follows that $\mathfrak{M}$ is a finite von Neumann algebra. Let $\mathfrak{R}$ be the set of operators affiliated with $\mathfrak{M}$ and having $\Omega$ in their domain. It follows from [5] that $\mathfrak{N}$ is a self-adjoint space and $\mathfrak{N M} \subseteq \mathfrak{M}$. This last statement follows because $\mathfrak{M N} \subseteq \mathfrak{N}$ and $\mathfrak{M M}=(\mathfrak{M M})^{*}$. If the definition of a spatial derivation is generalized to allow a mapping

$$
A \in D(\delta) \cong \mathfrak{M} \rightarrow \delta(A) \in \mathfrak{N}
$$

then the result of Theorem 1 is still valid. The proof of this more general result needs a slight extension of Lemma 5 of [1] to establish that the automorphism property is equivalent to the positivity preserving property

$$
(1+i \alpha H)^{-1} \mathfrak{M}_{+} \Omega \subseteq \overline{\mathfrak{M}_{+} \Omega}, \quad \alpha \in \mathbb{R} \backslash\{0\}
$$

and in the proof of Lemma 2 above $\delta(f(B))$ must be calculated directly in the vector state given by $\Omega$.

Acknowledgements. We are indebted to G. Gallavotti for several illuminating discussions concerning his joint work with M. Pulvirenti. We would also like to thank L. Zsido and S. L. Woronowicz for a number of useful discussions of finite von Neumann algebras.

\section{References}

1. Bratteli, O., Robinson,D.W.: Unbounded derivations of von Neumann algebras, Marseille preprint 75, P 733 (June 1975)

2. Gallavotti, G., Pulvirenti, M.: Classical KMS condition and Tomita-Takesaki theory. Commun. math. Phys. 46, $1-9$ (1976)

3. Kato, T.: Perturbation theory for linear operators. Berlin-Göttingen-Heidelberg: Springer 1966

4. Powers, R.: A remark on the domain of an unbounded derivation of a $C^{*}$-algebra. J. Funct. Anal. 18, 85-95 (1975)

5. Murray, F.J., von Neumann, J.: On rings of operators. Ann. Math. 37, 116-229 (1936)

Communicated by J.L. Lebowitz

Received July 24, 1975 
